\title{
Eine unscheinbare Arbeit aus den „Klinischen Monatsblättern“ von 1944
}

\section{An Inconspicuous Contribution in the “Klinische Monatsblätter” 1944}

Im Rahmen der Untersuchungen zu einem anderen Thema stieß der Autor bei der Durchsicht der Jahrgänge 19321945 der „Klinischen Monatsblätter für Augenheilkunde“ zufälligerweise auf die Arbeit „Sehphysiologische Untersuchungen an menschlichen Netzhäuten. 1. Die Farbsubstanzen“ von G.v. Studnitz [1] ( Abb. 1). Die Arbeit wirkt auf den ersten Blick „völlig harmlos“, da die Herkunft der untersuchten Augen nicht angegeben ist. Ihr Hintergrund offenbart sich aber bei genauer Analyse auch demjenigen, der sich mit den sehr vielfältigen Facetten der „Augenheilkunde im Nationalsozialismus“ [2] bisher nicht befasst hat. Da es sich bei den Untersuchungen um menschliche Netzhäute handelte, die Entnahme der Bulbi „unmittelbar post mortem bei rotem Dunkelkammerlicht“ erfolgte und vor allem ,in einer zweiten Versuchsserie die Dunkeladaptation bereits vor dem Exitus vorgenommen werden konnte“, ergibt sich zweifelsfrei, dass der Tod planbar war, es sich bei der „Ge- legenheit hierzu“ (Anmerkung: zur Untersuchung menschlicher Augen) also um Hinrichtungen handelte.

Gotthilft von Studnitz wurde 1908 in Kiel geboren. Nach Studium der Physiologie, Zoologie, Botanik und Geologie in Kiel und Breslau promovierte er 1930 in Kiel, wo er sich 1935 auch habilitierte. Ab 1936 war von Studnitz zunächst als Assistent in Halle/Saale tätig, 1937 trat er der NSDAP bei, 1941 wurde er „außerplanmäßiger Professor“, 1942 schließlich ordentlicher Professor für Zoologie und Direktor des zoologischen Instituts und Museums der Universität Halle [3]. Gotthilft von Studnitz war nach 1945 beruflich in Lübeck tätig. Er starb 1994 in Bad Schwartau bei Lübeck.

Wissenschaftlich beschäftigte sich von Studnitz vor allem mit den Sehpigmenten. 1941 erhielt er einen Forschungsauftrag von den Farbenwerken Wolfen, 1942 einen solchen vom Oberkommando der Marine. Letzterer diente dem Ziel, die Dunkeladaptation von (Marine-) Soldaten $\mathrm{zu}$ verbessern und Blendwirkungen $\mathrm{zu}$ vermindern. Der Auftrag war also eindeutig wehrmedizinisch motiviert. In Verfolgung der Forschungsprojekte trat von Studnitz Anfang 1944 an den Staatsanwalt in Halle heran mit der Bitte, Hinrichtungen „für wissenschaftliche Zwecke modifiziert" vornehmen zu lassen. Der Bitte wurde stattgegeben. Dieser Vorgang und das Schreiben von Studnitz' waren seit Längerem bekannt [2,4,5]. Nach Kenntnis des Autors war bisher aber nicht bekannt, ob und wo die Untersuchungsergebnisse publiziert wurden. Es waren zumindest zu einem wesentlichen Teil die „Monatsblätter“ mit der „unscheinbaren Arbeit von 1944“.

Stefan Töpel und Frank Tost aus Greifswald waren die ersten, die auf Untersuchungen an den Augen eines Hingerichteten hingewiesen haben [6,7]. Insbesondere Neuropathologen, Pathologen und Anatomen [8] haben bereits vor Jahren damit begonnen, die „zweifelhafte Herkunft" von Gewebeproben für morphologische Untersuchungen aus der NSZeit zu thematisieren und eine entsprechende Sichtung ihrer Sammlungen vorzunehmen. $\mathrm{Zu}$ berücksichtigen bleibt indes, dass die ethischen Standards im Wesentlichen erst nach 1945 und gerade als Antwort auf die NS-Medizinverbrechen formuliert wurden, und dass Hinrichtungen seinerzeit, selbst wenn sie oft aus höchst nichtigem Anlass erfolgten, „legal“ waren. Und so können wir nach derzeitigem Kenntnisstand noch nicht einmal

\section{LXII.}

Aus dem Zoologischen Institut der Universität Halle/Saale

\section{Sehphysiologische Untersuchungen an menschlichen Netzhäuten \\ 1. Die Farbsubstanzen \\ Von G.v. Studnitz \\ Mit 2 Textabbildungen*) und 2 Tabellen}

I.

Die Sehstoffe des Zapfenapparates sind nunmehr bei Vertretern aller Wirbeltierklassen nachgewiesen $\left.\left.\left.{ }^{1}\right)^{2}\right)^{3}\right)^{4}$ ); bezüglich ihrer Wirkungsbereiche ${ }^{5}$ ), Absorptionsmaxima und Lichtbleiche ${ }^{0}$ ) konnten sie beim Frosch, der Ringelnatter, dem Kücken

1) G. v. S t ud n i t z, Pflugers Arch. 1932, Bd. 230 , S. $615-638$

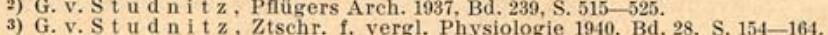

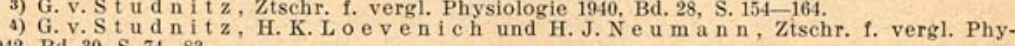
siol. 1942, Bd. 30, S. $74-83$. 3 , H. Wig g e r und H. K. L o eve n i c h, Pfügers Arch. 1944, Bd. 247 S. $353-356$.

6) H. K. Loevenich und G. v. Studnitz, Z. vergl. Physiol. 1943, Bd. 30, S. 167-180. *) Abbildungen gingen durch Feindeinwirkung verloren.
Abb. 1 Titel der Arbeit [1].

Abb. 2 Zusammenfassung der Ergebnisse [1]. 
ausschließen, dass (ophthalmo-) morphologische Untersuchungen an den Augen Hingerichteter bereits in der Weimarer Republik und im Kaiserreich, also schon vor 1933 und bei erheblich geringerer Hinrichtungsfrequenz, praktiziert wurden.

Angesichts der Herkunft der Augen dürfte und sollte die wissenschaftlich-nüchterne Zusammenfassung ( $\mathbf{A b b}$. 2) bei den heutigen Lesern Nachdenklichkeit hervorrufen. Festzuhalten bleibt, dass von „bedenklichen“ Arbeiten, die im Zeitraum 1933-1945 publiziert wurden, nicht nur „Graefes Archiv“ [6,7], sondern auch die „Klinischen Monatsblätter“ betroffen waren.

\section{Interessenkonflikt: Nein.}

\section{J. M. Rohrbach}

Department für Augenheilkunde,

Forschungsbereich Geschichte der Augenheilkunde/Ophthalmopathologisches

Labor, Eberhard-Karls-Universität Tübingen

\section{Literatur}

1 von Studnitz G. Sehphysiologische Untersuchungen an menschlichen Netzhäuten. 1. Die Farbsubstanzen. Klin Monatsbl Augenheilk 1944; 110: 617-620

2 Rohrbach JM. Augenheilkunde im Nationalsozialismus. Stuttgart: Schattauer; 2007

3 Im Internet: http://de.wikipedia.org/wiki/ Gotthilft_von_Studnitz; Stand: 15.01.2014

4 Klee E. Deutsche Medizin im Dritten Reich. Karrieren vor und nach 1945. Frankfurt/ Main: S. Fischer; 2001: 137-147

5 Rohrbach JM. Editorial zu „Vom Auge eines Hingerichteten“. Klin Monatsbl Augenheilk 2013; 230: 1257-1258

6 Töpel S, Tost F. Vom Auge eines Hingerichteten. Klin Monatsbl Augenheilk 2013; 230: 1259-1262

7 Krümmel $\mathrm{H}$. Die Nerven des menschlichen Ciliarkörpers. Ein Beitrag zur Neurohistolo- gie der glatten Muskulatur. Graefes Arch Ophthalmol 1938; 138: 845-865

8 Redies C, Hildebrandt S. Anatomie im Nationalsozialismus. Ohne jeglichen Skrupel. Dtsch Ärztebl 2012; 109: B-1968-B-1969

\section{Bibliografie}

DOI http://dx.doi.org/10.1055/s-0033-1360379

Online-publiziert: 8.4.2014

Klin Monatsbl Augenheilkd 2014; 231: 640-641

(c) Georg Thieme Verlag KG Stuttgart · New York . ISSN 0023-2165

\section{Korrespondenzadresse}

\section{Prof. Dr. Jens Martin Rohrbach}

Eberhard-Karls-Universität Tübingen

Department für Augenheilkunde, Forschungsbereich Geschichte der Augenheilkunde/

Ophthalmopathologisches Labor

Schleichstraße 6-12

72076 Tübingen

Tel.: $++49 /(0) 7071 / 2984761$

Fax: $++49 /(0) 7071 / 294762$

Martin.Rohrbach@med.uni-tuebingen.de

\section{License terms}

